\title{
EDITORIAL
}

\section{High-dose chemotherapy and autologous hematopoietic stem cell transplantation for metastatic breast cancer: a therapy whose time has passed}

\author{
Bone Marrow Transplantation (2006) 37, 985-987. \\ doi:10.1038/sj.bmt.1705366
}

Over the past two decades, the investigation of high-dose chemotherapy and autologous hematopoietic stem cell transplantation for breast cancer has followed the path of a roller-coaster ride. Initial results from numerous initial Phase II studies were remarkable: the response rate in patients with metastatic disease who underwent high-dose consolidation were impressively high, and a subset of up to $30 \%$ remained disease-free for a prolonged period. ${ }^{1-5}$ Larger prospective comparisons of conventional dose therapy to one or two cycles of high-dose therapy with transplant were initiated in the early 1990s but accrued slowly, with up to a decade from initiation to reporting of results. Reasons for the slow accrual included the hesitance of many third party payers to cover the clinical costs of patients enrolled on these trials, the biases of many physicians, and strident patient advocacy at times for and at times against the procedure. The first reports from randomized trials at a single institution in both early stage and metastatic disease showed dramatic survival benefits but were ultimately shown to be based on fraudulent data. ${ }^{6}$ In the interim, however, the treatment became almost standard-of-care, several US states enacted legislation requiring insurance companies to provide reimbursement, and many patients received high-dose therapy off protocol, reducing accrual to ongoing randomized comparisons. The results of those randomized studies were eventually presented beginning in 1999 but showed little benefit, and the number of procedures has now fallen from thousands yearly to only a few.

Six randomized studies have examined the effect of highdose chemotherapy in patients with metastatic disease: the North American ECOG/Philadelphia Bone Marrow Transplant Group PBT-1 Trial, the Canadian MA-16 study, the French PEGASE 03 and 04 studies, and two trials from Duke University, one of which appears in this issue of Bone Marrow Transplantation.

The PBT-1 Trial randomized 189 women with metastatic breast cancer and at least a partial response to initial chemotherapy to either a prolonged course of standard dose chemotherapy or a single 4-day infusion of high-dose cyclophosphamide, carboplatin, and thiotepa with autologous stem cell support. ${ }^{7}$ At the final analysis, the median overall survival was 24 months in the high-dose therapy group and 26 months in the standard dose group. The $95 \%$ confidence interval for the hazard ratio for the likelihood of overall survival was $0.53-1.17$, such that even accounting for the observed rate of noncompliance, the data were inconsistent with a relative benefit of greater than $24 \%$. Time to progression was also not different, with medians of 9.6 and 9.0 months in the high-dose and standard dose groups, respectively. Further analyses by extent of response to induction chemotherapy, estrogen-receptor status, or the presence of visceral metastases failed to identify a subgroup in which high-dose therapy was beneficial, but among patients older than 42 years, high-dose therapy appeared to be associated with worse overall survival. This study has been criticized for its choice of high-dose therapy (although STAMP V was the most common regimen in use at the time of the study's design), for randomizing only responding patients, and for using a control group actively treated with prolonged akylating agent therapy. Five year follow-up data continue to show no benefit, with less than $5 \%$ of patients in each arm free from progression. ${ }^{8}$

The Canadian MA-16 trial randomized 224 patients with metastatic breast cancer who had responded to four cycles of standard dose anthracycline- or taxane-based induction therapy. ${ }^{9}$ Patients who were hormone-receptor positive were not required to fail hormonal therapy to be eligible. The patients were randomized to receive $2-4$ additional cycles of standard dose chemotherapy or 1-2 additional cycles followed by high-dose cyclophosphamide, mitoxantrone, and carboplatin. The fatal toxicity rate associated with transplant was $6.3 \%$. With a median of 4 years of follow-up, high-dose therapy resulted in an improvement in progression-free survival (41 versus $28 \%$ at 1 year, $P=0.0143$ ) but not overall survival (31 versus $36 \%$ at 2 years, $P=0.9$ ).

The French PEGASE 03 study randomized 180 patients with metastatic breast cancer and at least a partial response after initial therapy with four cycles of fluorouracil, epirubicin, and cyclophosphamide (FEC100) to either observation or consolidation with one cycle of high-dose thiotepa and cyclophosphamide. ${ }^{10,11}$ Only 80 of the 89 patients randomized to high-dose therapy actually received the intended transplant, one of whom died of treatmentrelated toxicity. The high-dose therapy arm was nevertheless associated with an improved response rate $(92$ versus $59 \%, P=0.01)$ and improved disease-free survival at 1 year (46 versus $19 \%, P=0.0001$ ). Overall survival, however, was not statistically different at 3 years $(38 \%$ for the high-dose therapy arm versus $30 \%$ for the observation arm). 
PEGASE 04 was a small study with a similar structure that randomized 61 patients with metastatic breast cancer and at least a partial response after initial anthracycline-based chemotherapy. ${ }^{12}$ Patients received either maintenance standard dose chemotherapy or highdose mitoxantrone, cyclophosphamide, and melphalan with stem cell support. The study was stopped prematurely owing to poor accrual but found a benefit from high-dose therapy in time to progression (median 12 months versus 6 months, two-side $P$-value $<0.012$ ), with only a suggestion of an overall survival benefit (median 44.1 versus 19.3 months, two-sided $P$-value $<0.0588$ ).

Taken together, the results of these trials were disappointing, suggesting that an improvement in time to progression from high-dose therapy, if present, did not translate into an improvement in overall survival. Many reasons for these disappointing results have been suggested, including patient selection, choice of transplant and control regimens, and lack of statistical power. A nonrandomized study has compared registry patients who had received high-dose therapy with patients receiving standard dose therapy on CALGB protocols; this study was hampered by the need to control for imbalances in multiple known prognostic factors but suggested that any benefit of highdose chemotherapy would be restricted to a small number of patients and to the period of time greater than 3 years after transplant. ${ }^{13}$

Vredenburgh et al. at Duke University have now presented the results of two trials comparing immediate to delayed high-dose chemotherapy in specific subgroups of patients with metastatic breast cancer. A recently published trial enrolled 425 patients with metastatic or inflammatory breast cancer and randomized 100 who were able to achieve a complete response after 2-4 cycles of doxorubicin, fluorouracil, and methotrexate. ${ }^{14}$ Patients proceeded to either observation or consolidation with a single course of high-dose cyclophosphamide, cisplatin, and carmustine (also known as STAMP-1) with both bone marrow and peripheral blood stem cell support followed by radiation to soft-tissue metastatic sites. Patients who relapsed on the observation arm were able to receive high-dose therapy at the time of relapse. Event-free survival favored the immediate high-dose therapy arm (median 9.7 versus 3.8 months, $P=0.006$ ), but overall survival was similar in the two arms $(P=0.69)$. The overall transplant-related mortality rate was $8.6 \%$, including patients in the immediate transplant arm, patients transplanted at relapse, and patients transplanted after only a partial response to induction.

In this issue of Bone Marrow Transplantation, Vredenburgh et al. ${ }^{15}$ provide the final results from another randomized trial comparing immediate to delayed highdose therapy in the subgroup of breast cancer patients with bone-only metastases. They enrolled 85 patients with breast carcinoma metastatic to bone and no other sites (except the bone marrow) who had received no prior chemotherapy for metastatic disease but had failed at least one hormonal therapy if the malignant cells expressed estrogen or progesterone receptors. All patients received induction therapy with 2-4 cycles of doxorubicin, fluorouracil, and methotrexate, and the 69 patients without progression after induction therapy were randomized. The control arm received intensive consolidative radiation to all known sites of metastasis followed by observation until progression; high-dose therapy consisted of infusional cyclophosphamide and cisplatin followed by carmustine, with granulocyte colony-stimulating factor mobilized autologous peripheral blood stem cell support, then followed by the same intensive radiotherapy and observation. Patients in the control arm were offered the same high-dose regimen at the time of disease progression. The study was designed to have $80 \%$ power to detect a $50 \%$ reduction in hazard.

This study showed an improvement in time to progression but not overall survival. Immediate high-dose therapy was associated with an improvement in median event-free survival to 6 months from 4.3 months in the observation arm, a result that is highly statistically significant $(P<0.0001)$. The median overall survival in the immediate high-dose therapy group was numerically better than that in the delayed transplant group (2.97 versus 1.81 years) but with a high probability that the difference could have been due to chance alone $(P=0.144)$. The rate of transplantrelated mortality was approximately $10 \%$.

With these reports, Vredenburgh and co-workers have presented important long-term follow-up data on two subgroups of patients with metastatic breast cancer in which the efficacy of high-dose therapy has previously been poorly characterized. They and their patients are to be congratulated for completing and reporting these trials.

However, several aspects limit the clinical utility of these data. The control arms in these studies clearly do not represent the standard of care for the treatment of any group of patients with metastatic breast cancer; combination chemotherapy may be indicated for some patients, but intensive consolidation radiotherapy and prolonged periods of observation without therapy are unconventional approaches. The ability of additional chemotherapy to delay progression compared to observation is not surprising and would likely be expected for standard doses as well. Moreover, the benefit to a patient of delaying (possibly asymptomatic) radiologic progression with the use of a relatively toxic high-dose regimen with a $10 \%$ transplantrelated mortality rate is questionable. A statistically relevant improvement in overall survival of the magnitude suggested in this report of patients with bone-only metastases would be a clear advance in patient outcomes, but larger studies would be necessary to show this benefit if it is indeed present.

The authors note that all of their long-term survivors received high-dose therapy at some point during the study. This observation highlights the dangers of selection bias in the interpretation of uncontrolled trials: by the structure of this protocol, patients who did not receive high-dose therapy had progressive disease or refused therapy, and patients who were able to undergo transplant clearly had less aggressive disease and probably better performance status at the time of transplant. It therefore remains unclear whether the survivors did well because they received highdose therapy or they received high-dose therapy because they were already doing well.

The history of high-dose therapy for breast cancer has been dramatic, encompassing fraudulent results, legislative 
battles, and a familiar sequence of events in which impressive initial results seen in uncontrolled Phase II trials have not been reproduced in larger randomized studies. The current studies by Vredenburgh and coworkers do not provide compelling evidence to change these impressions. In 2006, little role remains for high-dose chemotherapy and autologous stem cell support in the treatment of breast carcinoma. Newer agents, such as taxanes and monoclonal antibodies, have shown benefits in both the adjuvant and metastatic settings without large increases in toxicity and certainly without the upfront mortality that has plagued certain regimens used in autologous transplantation. The toxicity of high-dose regimens and the lack of overall survival benefit make autologous transplant unlikely to be a better platform for the addition of novel agents when compared to standard doses of chemotherapy. We must therefore conclude that the current era belongs to less toxic novel and targeted agents and that the time of high-dose therapy and autologous stem cell transplant for breast cancer has passed - at least for now.

DT Vogl and EA Stadtmauer Abramson Cancer Center, University of Pennsylvania School of Medicine, Philadelphia, PA, USA E-mail: stadtmae@uphs.upenn.edu

\section{References}

1 Peters WP, Shpall EJ, Jones RB, Olsen GA, Bast RC, Gockerman JP et al. High-dose combination alkylating agents with bone marrow support as initial treatment for metastatic breast cancer. J Clin Oncol 1988; 6: 1368-1376.

2 Williams SF, Mick R, Desser R, Golick J, Beschorner J, Bitran JD. High-dose consolidation therapy with autologous stem cell rescue in stage IV breast cancer. J Clin Oncol 1989; 7: 18241830.

3 Antman K, Ayash L, Elias A, Wheeler C, Hunt M, Eder JP et al. A phase II study of high-dose cyclophosphamide, thiotepa, and carboplatin with autologous marrow support in women with measurable advanced breast cancer responding to standard-dose therapy. J Clin Oncol 1992; 10: 102-110.

4 Williams SF, Gilewski T, Mick R, Bitran JD. High-dose consolidation therapy with autologous stem-cell rescue in stage IV breast cancer: follow-up report. J Clin Oncol 1992; 10: 1743-1747.

5 Kennedy MJ, Beveridge RA, Rowley SD, Gordon GB, Abeloff MD, Davidson NE. High-dose chemotherapy with reinfusion of purged autologous bone marrow following doseintense induction as initial therapy for metastatic breast cancer. J Natl Cancer Inst 1991; 83: 920-926.

6 Weiss RB, Gill GG, Hudis CA. An on-site audit of the South African trial of high-dose chemotherapy for metastatic breast cancer and associated publications. J Clin Oncol 2001; 19: 2771-2777.

7 Stadtmauer EA, O’Neill A, Goldstein LJ, Crilley PA, Mangan $\mathrm{KF}$, Ingle JN et al. Conventional-dose chemotherapy compared with high-dose chemotherapy plus autologous hematopoietic stem-cell transplantation for metastatic breast cancer. $N$ Engl J Med 2000; 342: 1069-1076.

8 Stadtmauer EA, O'Neill A, Goldstein LJ, Crilley PA, Mangan $\mathrm{KF}$, Ingle JN et al. Conventional-dose chemotherapy compared with high-dose chemotherapy (HDC) plus autologous stem-cell transplantation (SCT) for metastatic breast cancer: 5year update of the 'Philadelphia Trial' (PBT-1). Am Soc Clin Oncol 2002 (abstract \#169).

9 Crump M, Gluck S, Stewart D, Levine M, Pritchard K, Kirkbride $\mathrm{P}$ et al. A randomized trial of high-dose chemotherapy (HDC) with autologous peripheral blood stem cell support (ASCT) compared to standard therapy in women with metastatic breast cancer: a National Cancer Institute of Canada (NCIC) Clinical Trials Group study. Am Soc Clin Oncol 2001 (abstract \#82).

10 Roche H, Viens P, Biron P, Lotz JP, Asselain B. High-dose chemotherapy for breast cancer: the French PEGASE experience. Cancer Control 2003; 10: 42-47.

11 Biron P, Durand M, Roche H, Delozier T, Battista C, Fargeot $\mathrm{P}$ et al. High dose thiotepa (TTP), cyclophosphamide (CPM) and stem cell transplantation after 4 FEC 100 compared with 4 FEC alone allowed a better disease free survival but the same overall survival in first line chemotherapy for metastatic breast cancer. Results of the PEGASE 03 French Protocole. Am Soc Clin Oncol 2002 (abstract \#167).

12 Lotz JP, Cure H, Janvier M, Asselain B, Morvan F, Legros M et al. High-dose chemotherapy with haematopoietic stem cell transplantation for metastatic breast cancer patients: final results of the French multicentric randomised CMA/PEGASE 04 protocol. Eur J Cancer 2005; 41: 71-80.

13 Berry DA, Broadwater G, Klein JP, Antman K, Aisner J, Bitran $\mathbf{J}$ et al. High-dose versus standard chemotherapy in metastatic breast cancer: comparison of cancer and leukemia group B trials with data from the autologous blood and marrow transplant registry. J Clin Oncol 2002; 20: 743-750.

14 Vredenburgh JJ, Coniglio D, Broadwater G, Jones RB, Ross M, Shpall EJ et al. Consolidation with high-dose combination alkylating agents with bone marrow transplantation significantly improves disease-free survival in hormone-insensitive metastatic breast cancer in complete remission compared with intensive standard-dose chemotherapy alone. Biol Blood Marrow Transplant 2006; 12: 203.

15 Vredenburgh JJ, Madan B, Coniglio D, Ross M, Broadwater $\mathrm{G}$, Niedzwiecki D et al. A Randomized phase III comparative trial of immediate consolidation with high dose chemotherapy and autologous peripheral blood progenitor cell support compared to observation with delayed consolidation in women with metastatic breast cancer and only bone metastases following intensive induction chemotherapy. Bone Marrow Transplant 2006; 37: 1009-1015. 